\title{
Real clocks and the Zeno effect
}

\author{
Inigo L. Egusquiza ${ }^{1}$ and Luis J. Garay ${ }^{2}$ \\ ${ }^{1}$ Department of Theoretical Physics, The University of the Basque Country, 644 Posta Kutxa, 48080 Bilbao, Spain \\ ${ }^{2}$ Institute of Mathematics and Fundamental Physics, CSIC, c/ Serrano 121, 28006 Madrid, Spain
}

(Received 30 January 2003; published 13 August 2003)

\begin{abstract}
Real clocks are not perfect. This must have an effect in our predictions for the behavior of a quantum system, an effect for which we present a unified description, encompassing several previous proposals. We study the relevance of clock errors in the Zeno effect and find that generically no Zeno effect can be present (in such a way that there is no contradiction with currently available experimental data). We further observe that, within the class of stochasticities in time addressed here, there is no modification in emission line shapes.
\end{abstract}

DOI: 10.1103/PhysRevA.68.022104

PACS number(s): 03.65.Ta

\section{INTRODUCTION}

The problem of understanding time observables in quantum mechanics has a long and protracted history [1]. One of the key observations in the process of a better formulation of time quantities was carried out by Misra and Sudarshan [2], investigating how the measurement of lifetimes could be affected by frequent probes into the evolution of the system under study. It should be pointed out that Misra and Sudarshan placed their work in the context of time observables, making explicit connections to the problem of time of arrival, as discussed by Allcock [3-5].

The result of Misra and Sudarshan that a continuously observed unstable particle would never decay was associated by them with the name of Zeno of Elea, and it is under this title that the effect or paradox is currently known. In fact, the effect had been pointed out before in different forms [6,7], as was indicated by Chiu, Sudarshan, and Misra [8]. At any rate, the number of papers referring to the Zeno effect or paradox increased substantially after 1977, and even more so after the crucial experiment of Itano et al. [9] (for a review of research in the topic up to 1997, with the corresponding bibliography, see Ref. [10]; a more up-to-date review of bibliography can be found in Ref. [11]).

Another intriguing aspect of time in quantum mechanics is related to decoherence and decoherence rates, both in a general sense, and more specifically as a source of decoherence. Milburn [12] proposed a simple modification of quantum dynamics in which the system does not evolve continuously under unitary evolution: it undergoes a sequence of identical unitary transformations, which take place or not according to a Poisson distribution (i.e., the probability that there be $n$ such transformations in a time interval $t$ is given by a Poisson distribution). This proposal leads to decoherence, while at the same time it conserves energy (a feature lacking in previous models of intrinsic decoherence, such as the well-known one of Ghirardi, Rimini, and Weber [13]).

A different aspect of stochasticity in time was put forward in Ref. [14]: the fact that our clocks are not perfect implies that incoherent superpositions of states at different instants of time are going to be necessary to account for the state observed at a given clock instant. The requirements for a clock to be considered good were examined and formalized, leading to a claim of uniqueness for the effective description of good clocks, in terms of a stationary, Gaussian, and Markovian stochastic process.

Bonifacio also proposed a generalization of Liouville's equation just by requiring that time be an stochastic variable and demanding that a different form of stationarity hold [15] [see below, Eq. (3) and the surrounding discussion, for the expression of this property]. He further posed a claim of uniqueness for the probability distribution that encoded stochasticity in time, which he asserted was the $\Gamma$ distribution.

All these three proposals shared the result that, under adequate approximations, the systems behaving according to them would actually follow an evolution equation for the density matrix of the form

$$
\dot{\rho}(t)=-i[H, \rho(t)]-\frac{\kappa^{2}}{\vartheta}[H,[H, \rho(t)]]
$$

where we have set $\hbar$ to 1 , as we do in the following, $H$ is the Hamiltonian of the system under consideration, described with the density matrix $\rho$ at clock time $t$, and $\kappa$ and $\vartheta$ are constants with dimensions of time. In the formalism of Ref. [12], the quotient $\kappa^{2} / \vartheta$ is associated with the intrinsic time step of the unitary evolutions; in Ref. [14] $\kappa^{2} / \vartheta^{2}$ stands for the strength of the correlation function of relative errors (the rates of increase or decrease of the clock error at different clock times), whereas $\vartheta$ is the correlation time for those relative errors; to be complete we mention that the combination $\kappa^{2} / \vartheta$ is equivalent to the "chronon" of Ref. [15].

In fact, this kind of master equation was also known from the analysis of heat baths coupled to the system by a term of the form $H \Gamma$, where $H$ is the system's Hamiltonian and $\Gamma$ some bath operator (see, for instance Ref. [16], Sec. 2.3). The explicit connection between the proposal that quantum (and classical) systems evolve according to nonideal clocks, on the one hand, and the heat bath language, on the other, was shown in Ref. [14].

In a recent paper [17], Adler, using the language of Itô calculus, considered together both Zeno's effect and Eq. (1), with the result that the Zeno effect would be washed out by the new time scale $\kappa^{2} / \vartheta$. Given the results of Facchi and Pascazio (and others) $[18,11]$, it is rather surprising that the Zeno effect disappears no matter what the value of the new time scale is. Even more, if this equation is the result of a 
coupling between the system being considered and the heat bath, the total system (system plus bath) will of necessity present Zeno's effect.

We should mention, for the sake of completeness, that the idea of errors in time measurements seems to be cropping up in several other contexts by various authors (for a couple of recent examples, see Ref. [19,20]). The motivation behind most of those efforts seems to be either a desire to understand decoherence better or the search for modifications of the ordinary quantum axioms regarding evolution. An example of this latter approach is to be found in the series of papers [21,22]; notice that the specific form of nonlocality in time put forward in those papers leads to radical modification of the line shape, contrary to the results of Adler's and our own for nonlocality due to randomness in the measurement of time.

The purpose of this paper is thus threefold: (1) to present a general formalism that accounts for various different proposals in a unified manner and explains the centrality of the approximations leading to Eq. (1); (2) to examine whether Zeno's effect is indeed generically washed away by the mere fact that time should be considered as stochastic or whether the result of Adler's does not indeed extend to more general situations; (3) to resolve the different claims of uniqueness which seem contradictory.

\section{UNIFIED FORMULATION}

A good starting point is given by the initial stages of Bonifacio's formulation [15], which we present here with a notation and interpretation closer to that of Ref. [14]. Let $\rho_{S}(s)$ represent the density matrix of system $S$ at Schrödinger's ideal time $s$; that is to say, $\rho_{S}$ evolves according to von Neumann's equation

$$
\frac{d}{d s} \rho_{S}(s)=-i\left[H, \rho_{S}(s)\right]=-i \mathcal{L} \rho_{S}(s),
$$

where $\mathcal{L}$ is the Liouvillian (super)operator.

This evolution equation is used to make predictions about the outcomes of experiments that we can describe in the following manner: after a preparation stage, in which we make sure that we have set the system in a well-defined initial state, we let the system evolve a time interval $t$ that we measure with our clock. At that instant, we measure the value of some observable of the system. Our predictions, in general, will not concern the actual value measured in a single instance of the experiment; they will rather provide us with probability distributions, which will be checked by many repetitions of the experimental procedure.

However, any clock we might use will have intrinsic uncertainties and the ideal time elapsed in each instance of the experiment will be different, even if we insist on always measuring the same time interval with our clock. We should then realize that the predictions we are required to provide must be predictions in clock time $(t)$, not in ideal time $(s)$, that is, we must have a way of computing probability distributions for all observables at time $t$. Through the standard arguments, this means that we can encode our predictions in a density matrix at clock time $t, \rho(t)$.

The actual observed/predicted density matrix at clock time $t$ will be given by a superposition of the density matrices at different ideal times, under the assumption that, for different realizations of our experiment, the same reading $t$ of our clock corresponds to different ideal time intervals $s$ having elapsed since the preparation of the initial state. Let us describe this assumption by a probability density for those ideal times $s$ at a fixed $t, P(t, s)$ : the actual density matrix $\rho(t)$ will be computed as

$$
\rho(t)=\int_{0}^{\infty} d s P(t, s) \rho_{S}(s)=\int_{0}^{\infty} d s P(t, s) e^{-i s \mathcal{L}} \rho_{S}(0) .
$$

Here, one simple underlying assumption is that the preparation instant be labeled by $s=0$ and $t=0$. Another assumption is that we know for certain that, at the instant we let the system go, the state is indeed always the same.

Furthermore, we suppose that the clock goes forward in ideal time, which entails that the support of $P(t, s)$ be over negative $s$; by the very definition of the probability density, which is to be used to predict the results of experiments using real clocks, the clock time interval $t$ is of necessity positive. Note that a simple way of modifying the assumption of positive ideal time flow of the clock would be to allow $s$ to be negative. When we introduce later $\Pi_{\mathrm{G}}(t, k)$, in Eq. (13) below, we shall actually relax this condition on $s$, as explained at that point.

By the manner in which we have justified the introduction of $P(t, s)$, we have already demanded that it be a probability distribution. However, an alternative way of arriving at the same concept would be to assume Eq. (2) and that $\rho(t)$ indeed be a density matrix (as is to be expected from the general arguments of Gleason's theorem-see the strengthened version provided by Busch [23]). It would follow that $P(t, s)$ would have to be positive and normalized to 1 when integrated over $s$,

$$
\int_{0}^{\infty} d s P(t, s)=1
$$

There is yet another important property that this probability density should fulfill: the way in which errors could accumulate should be independent of the instant of time at which we have started the clock. At least it should be so for good clocks; the way new errors are produced should be independent of the error up to that instant. Note, however, that the error at one clock time $t+d t$ does depend on the error at time $t$. If this one is very big, it is very unlikely that at a later clock time the error could be zero. Therefore, the stationarity requirement we are now discussing cannot be understood as stationarity for the errors in time measurement, but rather as stationarity in the buildup of errors.

In order to obtain a mathematical statement of this stationarity requirement, consider the following setup: a system is prepared in an initial state $\rho_{S}(0)$ and is then evolved a clock time interval $t_{1}$. The state at this clock time is now 


$$
\rho\left(t_{1}\right)=\int_{0}^{\infty} d s_{1} P\left(t_{1}, s_{1}\right) e^{-i s_{1} \mathcal{L}} \rho_{S}(0) .
$$

By whichever means, the state is then frozen, and then we let it evolve a further time interval $t_{2}$. This is another way of saying that we repeat the evolution, but now for a different time interval, and with an initial state that is now $\rho\left(t_{1}\right)$. The end result would be the density matrix

$$
\begin{aligned}
\rho\left(t_{1}+t_{2}\right)= & \int_{0}^{\infty} d s_{2} P\left(t_{2}, s_{2}\right) e^{-i s_{2} \mathcal{L}} \rho\left(t_{1}\right) \\
= & \int_{0}^{\infty} d s_{2} P\left(t_{2}, s_{2}\right) e^{-i s_{2} \mathcal{L}} \\
& \times \int_{0}^{\infty} d s_{1} P\left(t_{1}, s_{1}\right) e^{-i s_{1} \mathcal{L}} \rho_{S}(0) .
\end{aligned}
$$

On the other hand, that freezing of the state need not be real, it is simply a tool of our imagination, and the density matrix at the end must be given by

$$
\rho\left(t_{1}+t_{2}\right)=\int_{0}^{\infty} d s P\left(t_{1}+t_{2}, s\right) e^{-i s \mathcal{L}} \rho_{S}(0) .
$$

This entails the following condition on the probability density $P$ :

$$
P\left(t_{1}+t_{2}, s\right)=\int_{0}^{s} d s^{\prime} P\left(t_{2}, s-s^{\prime}\right) P\left(t_{1}, s^{\prime}\right),
$$

which is the mathematical expression of the stationarity requirement.

In order to solve this functional equation, it is useful to use Fourier transforms. Define

$$
\Pi(t, k)=\int_{0}^{\infty} d s P(t, s) e^{i k s}
$$

Note for further use the following set of facts: since $P$ is a probability distribution for the stochastic variable $s$, it follows that $\Pi(t, 0)=1$ (remember that $s$ is stochastic with respect to the clock time which we measure); since $P$ has support only on the positive half line (as we are currently assuming) $\Pi(t, k)$ will have singularities only on the lower half of the complex $k$ plane. If all moments in $s$ of the probability distribution $P$ existed, then $\Pi(t, k)$ would be analytic at $k=0$.

By means of this definition, Eq. (3) is transformed into the algebraic equation

$$
\Pi\left(t_{1}+t_{2}, k\right)=\Pi\left(t_{1}, k\right) \Pi\left(t_{2}, k\right),
$$

with the solution

$$
\Pi(t, k)=[g(k)]^{t / \lambda},
$$

where the dimensionful parameter $\lambda$ has been introduced to adimensionalize the exponent, and $g(k)$ is an arbitrary function. Thus, $\lambda$ and $g(k)$ fully determine the characteristic function $\Pi(t, k)$, and consequently, the probability densities $P(t, s)$. The physical significance of $\lambda$ and $g(k)$ will become clear soon. For the time being, it is worth noticing that $g(k)$ is the characteristic function of the probability density $P(t, s)$ at time $t=\lambda$, and that changes in the parameter $\lambda$ can be compensated by a change in the function $g(k)$ as follows. Let $g_{\lambda}(k)$ be an arbitrary function that determines $\Pi(t, k)$ together with the parameter $\lambda$, that is to say, under the condition $\Pi(\lambda, k)=g_{\lambda}(k)$. It is then straightforward to see that the same distribution $\Pi(t, k)$ can be described in terms of a new function $g_{\mu}(k)$ and parameter $\mu$, such that $g_{\lambda}(k)$ $=\left[g_{\mu}(k)\right]^{\lambda / \mu}$.

The requirement that $\Pi(t, k)$ be the Fourier transform of a probability density, with the definition above, implies that $g(0)=1$. Similarly, the condition on the location of the singularities also applies to $g(k)$. Additionally, if we make the further hypothesis that all $(s)$ moments of the density $P(t, s)$ exist, this would entail that $g(k)$ would have to be analytic at $k=0$. It is also clear that $g(k) \rightarrow 0$ on the real line as $|k|$ $\rightarrow \infty$, if $P(t, s)$ is a continuous density.

Assuming the existence of the first two moments (in $s$ ) of the distribution $P(t, s)$, we can make the following interesting statements derived from the form of $\Pi(t, k)$ expressed in Eq. (5). The expectation value of $s$ is given by

$$
\langle s\rangle=\int_{0}^{\infty} d s P(t, s) s=-\left.i \partial_{k} \Pi(t, k)\right|_{k=0}=\frac{-i g^{\prime}(0)}{\lambda} t,
$$

where we have taken into account that $g(0)=1$. Observe that the expectation value is proportional to $t$ and that it is indeed exactly $t$ if $\lambda=-i g^{\prime}(0)$. It is immediate to appreciate that the ratio $-i g^{\prime}(0) / \lambda$ measures the systematic drift of the expected values of time. The systematic drift can be eliminated by recalibration of the clock, identified by its characteristic function, and, indeed, the ratio $-i g^{\prime}(0) / \lambda$ is invariant under the transformations $\lambda \rightarrow \mu$ above, and pertains exclusively to the function $\Pi(t, k)$ : if it is the case that $\lambda=-i g_{\lambda}^{\prime}(0)$, it is then true that $\mu=-i g_{\mu}^{\prime}(0)$. As to the variance, one can easily compute

$$
\Delta s^{2}=\int_{0}^{\infty} d s P(t, s)(s-\langle s\rangle)^{2}=\frac{1}{\lambda}\left[-g^{\prime \prime}(0)+g^{\prime}(0)^{2}\right] t,
$$

whence we see that $\Delta s \sim \sqrt{t}$ (with an adequate dimensionful proportionality constant).

The simplest function that fits those criteria [to recap: $g(0)=1$, analyticity at $k=0$, all singularities in the lower half plane, and $g(k) \rightarrow 0$ as $|k| \rightarrow \infty$ on the real line], and presents a singular point, is

$$
g_{\mathrm{B}}(k)=\frac{1}{1-i k \tau}
$$

where $\tau$ is a real number with dimensions of time. Notice that we are actually choosing a family of probability densities parametrized by $\lambda$, i.e., by the instant of time at which the characteristic function $\Pi(t, k)$ equals $g_{\mathrm{B}}(k)$. For each member of the family, as we shall see below, the meaning of 
the characteristic time $\tau$ will be slightly different. The subscript $B$ stands for Bonifacio: the probability density derived from this choice is

$$
P_{\mathrm{B}}(t, s)=\frac{1}{\tau} \frac{e^{-s / \tau}}{\Gamma(t / \lambda)}\left(\frac{s}{\tau}\right)^{t / \lambda-1},
$$

which is exactly the one put forward in Ref. [15]. The expectation value is $\langle s\rangle=\tau t / \lambda$ and the dispersion $\Delta s$ $=\tau \sqrt{t / \lambda}$. This means that among all the different members of this family of distributions, the one labeled by $\lambda=\tau$ is the only one for which there is no systematic drift in the expectation value of time; in which case $\tau$ gives the rate of growth of the dispersion of the successive probability densities $P(t, s)$.

Another simple alternative, with a very different analytic structure associated with periodicity, is given by

$$
g_{\mathrm{M}}(k)=\exp \left(e^{i k \tau}-1\right),
$$

where, again, $\tau$ is a real number with dimensions of time and the subscript $M$ now stands for Milburn. This function $g_{M}(k)$ is an entire periodic function, bounded on the real line (but, of course, not everywhere, in keeping with Liouville's theorem). On performing the inverse Fourier transform, one is led to the discrete probability density

$$
P_{\mathrm{M}}(t, s)=\sum_{n=0}^{\infty} \frac{1}{n !}\left(\frac{t}{\lambda}\right)^{n} e^{-t / \lambda} \delta(s-n \tau),
$$

which is precisely that proposed in Ref. [12] [Eq. (2.7); in order to make the identification between this formulation and the original one, it is convenient to use the construction of the following section]. This discrete probability density has the interpretation provided in that paper: the probability that there be $n$ identical unitary transformations of the density matrix, $\exp (-i \tau \mathcal{L}) \rho(0)$ in a time interval $t$ is given by the Poisson distribution. The expectation value $\langle s\rangle$ and the dispersion are given by the same expressions as those for $g_{\mathrm{B}}(k)$.

The two $g$ functions presented above do not, of course, exhaust all possible alternatives. Just as an example, consider

$$
g_{\mathrm{a}}(k)=\frac{1}{(1-i k \tau)(1-i k \sigma)},
$$

where both $\tau$ and $\sigma$ are positive time quantities (the subindex $a$ stands for "alternative"). Note that if $\tau$ and $\sigma$ are equal the resulting probability would be $P_{\mathrm{B}}(2 t, s)$, which could also be phrased as $P_{\mathrm{B}}(t, s)$ by halving the value of $\lambda$. Without loss of generality, let $\tau>\sigma$. The resulting probability density is

$$
\begin{aligned}
P_{\mathrm{a}}(t, s)= & \theta(s) \frac{\sqrt{\pi}}{\Gamma(t / \lambda)} \frac{1}{\sqrt{\sigma \tau}}\left(\frac{s}{\tau-\sigma}\right)^{-1 / 2+t / \lambda} \\
& \times e^{-(\tau+\sigma) s / 2 \sigma \tau} I_{t / \lambda-1 / 2}\left(\frac{(\tau-\sigma) s}{2 \sigma \tau}\right),
\end{aligned}
$$

with $I_{\nu}(z)$ the modified Bessel function. This probability density serves as a counterexample to the uniqueness claim presented in Ref. [15]. It should be pointed out that we make no claim whatsoever to the greater physical significance of this distribution, as compared to $P_{\mathrm{B}}$, and they are to be evaluated according to their fitting whatever phenomena we would like to describe. The expectation value computes to be $\langle s\rangle=(\sigma+\tau) t / \lambda$, while the dispersion reads as $\Delta s$ $=\sqrt{\left(\tau^{2}+\sigma^{2}\right) t / \lambda}$.

\section{THE MASTER EQUATION}

Direct comparison of the second integral in Eq. (2) with the definition of $\Pi(t, k)$ tells us that we can write the averaging process leading to the observed density matrix at clock time $t$ in the form

$$
\rho(t)=\Pi(t,-\mathcal{L}) \rho_{S}(0)=e^{(t / \lambda) \ln [g(-\mathcal{L})]} \rho_{S}(0) .
$$

By using the eigenoperators of the Liouvillian, that is to say, operators of the form $|n\rangle\langle m|$, where $|n\rangle$ and $|m\rangle$ are eigenstates of the Hamiltonian, we can write the exact evolution of the components of the density matrix in that basis: let $\rho(t)$ be given by $\Sigma_{m, n} \rho_{n m}|n\rangle\langle m|$. The components of the observed density matrix at clock time $t$ are related to those at time 0 by

$$
\rho_{n m}(t)=\Pi\left(t, E_{m}-E_{n}\right) \rho_{n m}(0) .
$$

This formal exact solution is very useful when analyzing simple systems; however, it might be cumbersome in more involved situations, and some other simplifications and limits might come in handy. For this reason, let us rewrite Eq. (6) as a differential equation,

$$
\dot{\rho}(t)=\frac{1}{\lambda} \ln [g(-\mathcal{L})] \rho(t) .
$$

Since $g(0)=1$ and $g(k)$ must be analytic at $k=0$, Eq. (8) admits an expansion whose first terms will be

$$
\dot{\rho}(t)=\frac{1}{\lambda}\left(-g^{\prime}(0) \mathcal{L}-\frac{1}{2}\left[-g^{\prime \prime}(0)+g^{\prime}(0)^{2}\right] \mathcal{L}^{2}+\cdots\right) \rho(t),
$$

or, taking into account that this expansion is actually the expansion of the generating function of the cumulants,

$$
\dot{\rho}(t)=\left(-i \frac{\langle s\rangle}{t} \mathcal{L}-\frac{1}{2} \frac{\Delta s^{2}}{t} \mathcal{L}^{2}+\cdots\right) \rho(t) .
$$

In order for this expansion to be physically relevant it is clear that there must be either a renormalization of the energy or else the first coefficient of the Taylor expansion of $g(k)$ around $k=0$ must be given by $g^{\prime}(0)=i \lambda$. In other words, the clock must be such that it adequately tracks ideal time, with no rescaling being necessary. Hence, here onwards we shall assume that the statement $g^{\prime}(0)=i \lambda$ does indeed hold [which entails a relation among the parameter $\lambda$ and the characteristic times appearing in $g(k)$ ]. It would be also pertinent to have some explanation for the validity of 
the expansion, which both for $g_{\mathrm{B}}(k)$ and $g_{\mathrm{M}}(k)$ reads, to second order in $k$, and making use of $\lambda=\tau$ [which is the specific form of the condition $\left.g^{\prime}(0)=i \lambda\right]$,

$$
\begin{aligned}
g_{\mathrm{B}, \mathrm{M}}(k) & =1+i \tau k-\tau^{2} k^{2}+O\left(\tau^{3} k^{3}\right) \rightarrow \dot{\rho}(t) \\
& =\left(-i \mathcal{L}-\frac{\tau}{2} \mathcal{L}^{2}+\cdots\right) \rho(t) .
\end{aligned}
$$

These examples lead us to understand expansion (10) as valid if the characteristic time of evolution (whose inverse gives us the characteristic expansion scale of $\mathcal{L}$ ) is much larger than the time constants that appear in the definition of the $g$ functions. In fact, those time constants, such as $\tau$ [or $\sigma$ and $\tau$ for $g_{\mathrm{a}}(k)$ ], characterize either the period [in cases such as $\left.g_{\mathrm{M}}(k)\right]$ or the closest singularities to the point $k=0$, so it is sensible to expect that this expansion will only be valid for characteristic evolution times larger than them, under the further demand that there is no resonant effect. It is important to notice that the characteristic evolution times are determined by the Hamiltonian of the system and by the initial condition, as follows. Since the Hamiltonian is usually semibounded and not bounded, the Liouvillian superoperator, whose eigenvalues are the differences of energy among energy eigenstates, is unbounded. However, if only a restricted set of energy eigenstates contribute to the initial state, the Liouvillian is bounded for that initial state and for all later states evolved from that one. It is bounded by the largest energy difference. We have previously demanded that $g(k)$ be analytic at $k=0$, so that the moments of the distribution $P(t, s)$ exist; it follows that there exists a $k_{c}$ radius of convergence. If the largest energy difference that comes into play is smaller than $k_{c}$, then the expansion above is convergent, and the approximation given by the truncation to the first two terms of the Taylor expansion is well controlled.

Notice that even if the Taylor expansion is well controlled some phenomena could be out of the scope of the approximation, in the case of periodic functions $g(k)$. In fact, Milburn [12] provided an explicit example of the breaking down of the expansion due to a resonant effect, by examining the average value of an oscillator and noticing that some frequencies would lead to the freezing of the evolution of the oscillator, namely, the harmonics of the evolution frequency $2 \pi / \tau$. Indeed, one should use in Eq. (7)

$$
\Pi_{\mathrm{M}}\left(t, E_{m}-E_{n}\right)=\exp \left[\frac{t}{\tau}\left(e^{i \tau\left(E_{m}-E_{n}\right)}-1\right)\right],
$$

leading to freezing of the element $\rho_{n m}(t)$ of the density matrix if $E_{m}-E_{n}=2 \pi l / \tau$, with $l$ an entire number, i.e., in the presence of a resonance among the characteristic times of system and real clock.

\section{GAUSSIAN WEIGHTS}

Having characterized the evolution equation (1) as a two term approximation of the whole family of evolutions (6), which fulfill the stationarity constraint (3), we should now examine to what extent the stationarity condition holds under the master equation (1). In order to do that, consider the more generic set of master equations

$$
\dot{\rho}(t)=h(t,-\mathcal{L}) \rho(t),
$$

where $h$ is a function fulfilling a number of conditions: to preserve the trace of the density matrix under this evolution, it is necessary that $h(t, 0)=1$ for all times; demanding that

$$
\int_{0}^{t} d s \operatorname{Re}[h(s, k)] \leqslant 0,
$$

for all $t$ and (real) $k$ is a sufficient condition to guarantee positivity.

In this case, the solution to the generalized master equation (11) is formally given by

$$
\rho(t)=\exp \left[\int_{0}^{t} d s h(s,-\mathcal{L})\right] \rho(0),
$$

whence we recover the description of Eq. (2), with the probability density computed as

$$
P_{h}(t, s)=\int_{-\infty}^{\infty} \frac{d k}{2 \pi} e^{-i k s} \exp \left[\int_{0}^{t} d s h(s, k)\right] .
$$

As an example, consider ordinary von Neumann evolution, associated with $h_{\mathrm{vN}}(t,-\mathcal{L})=-i \mathcal{L}$. It leads to $P_{\mathrm{vN}}(t, s)$ $=\delta(t-s)$, that is to say, to the identification of the appropriate clock as a perfect one, which keeps perfect track in clock time $t$ of the elapsed ideal time $s$.

Let us now consider the dephasing master equation (1), which is associated with

$$
h_{\text {m.e. }}(t, k)=i k-\frac{\kappa^{2}}{\vartheta} k^{2},
$$

and thus leads to

$$
P_{\text {m.e. }}(t, s)=\sqrt{\frac{\vartheta}{4 \pi \kappa^{2} t}} e^{-\vartheta(s-t)^{2} / 4 \kappa^{2} t} .
$$

It is readily seen that this probability density does indeed satisfy the stationarity requirement. However, it has support over negative values of $s$, not just on the positive half line.

It should be noticed that this is the unique Gaussian probability distribution (up to changes in parameters) that fulfills the stationarity requirement, which keeps exactly form (4) even if the support of $P(t, s)$ extends to the whole real line. Notice that the references to Gaussianity and support of the distribution concern the ideal time variable $s$.

More generally, we can characterize as stationary the evolutions in which $h(t, k)$ is in fact independent of $t$. In this manner, we see that the condition of stationarity stated above in terms of the probability densities is fully equivalent to the more standard requirement of the evolution being associated with a semigroup. Denoting $h(t, k)$ by $\ln [g(k)]$ if it is indeed independent of $t$, we see that the formalism presented in the previous sections goes through with the minor difference that 
the support of functions of $s$ extends to the whole real line, and that $g(k)$ is no longer required to be analytic in the whole upper half plane. The generator of the evolution semigroup is thus $\ln [g(-\mathcal{L})]$.

The meaning of the negative values of $s$ for a stationary distribution is that the clock can go backwards in ideal time, but, since it is for us a clock, we only perceive it as going forward. In other words, we organize the experiment according to the clock, so, by definition, we correlate measurement instants with a particular reading of the clock. If the clock were to go backwards, we could have marked the desired reading of the clock, which is a positive value of $t$, as having been reached before (in ideal time) the clock had started. This looks like a rather undesirable characteristic for a clock, and indeed in Ref. [14] it was characterized as a breakdown of "good" causality. On the other hand, if the characteristic error scale of the clock $\left(\kappa^{2} / \vartheta\right.$ in the Gaussian stationary example above) is extremely small when compared to the characteristic evolution of the system being analyzed, noncausal behavior will be unimportant.

There is a completely different reason for suspecting that the probability distribution (12) could have some nonphysical aspect to it. Consider the stochastic sequence of relative errors $\alpha(t)$. Formally, this is defined through the Langevin equation

$$
\frac{d s}{d t}=1+\alpha(t)
$$

Notice that the restriction that the probability density $P(t, s)$ should be zero for negative values of $s$ translates in this language to the (formal) requirement that $\alpha(t)$ be bigger than -1 at all times. However, this is not the only suspect aspect of this Langevin equation: distribution (12) corresponds to Gaussian white noise, that is to say, to the Gaussian stochastic sequence characterized by

$$
\langle\alpha(t)\rangle=0,\left\langle\alpha(t) \alpha\left(t^{\prime}\right)\right\rangle=\frac{\kappa^{2}}{\vartheta} \delta\left(t-t^{\prime}\right),
$$

for all $t$ and $t^{\prime}$. This suffers from the well-known shortcomings of white noise, namely, unphysicality of the infinite variance. Nonetheless, we have obtained this distribution as a generic limit of a wide class of possible distributions and, therefore, of a wide class of stochastic error sequences.

As a matter of fact, the notation used up to now $\left(\kappa^{2} / \vartheta\right.$ instead of a single quantity with dimensions of time) was designed with exactly this problem in mind. Consider an alternative Gaussian stationary stochastic sequence, characterized by

$$
\langle\alpha(t)\rangle=0,\left\langle\alpha(t) \alpha\left(t^{\prime}\right)\right\rangle=c\left(t-t^{\prime}\right) .
$$

This leads to a generating function

$$
\Pi_{\mathrm{G}}(t, k)=e^{i k t} e^{-k^{2} f(t)},
$$

where

$$
f(t)=\frac{1}{2} \int_{0}^{t} d t_{1} \int_{0}^{t} d t_{2} c\left(t_{1}-t_{2}\right) .
$$

The effective support of the correlation function $c(t)$ will be characterized by the correlation time $\vartheta$, while the maximum value $c(0)$ will be an adimensional number, $\kappa^{2} / \vartheta^{2}$. For large values of $t, f(t)$ will then be approximately proportional to $\kappa^{2} t / \vartheta$. We thus see that the distribution $P_{\text {m.e. }}(t, s)$ and its concomitant dephasing master equation are approximations in two different ways: on one hand, it is required that the characteristic evolution time of the system being studied be much smaller than the characteristic scale $\kappa^{2} / \theta$, so that the approximation of $\Pi(t,-\mathcal{L})$ can make sense; and, on the other, the elapsed time $t$ must be bigger than the relative errors' correlation time $\vartheta$.

As an specific example of nonwhite Gaussian noise, that will be adequately described by white noise for $t$ larger than $\vartheta$, consider the Ornstein-Uhlenbeck process, characterized by

$$
\langle\alpha(t)\rangle=0,\left\langle\alpha(t) \alpha\left(t^{\prime}\right)\right\rangle=\frac{\kappa^{2}}{\vartheta^{2}} e^{-\left|t-t^{\prime}\right| / \vartheta} .
$$

The function $f(t)$ would be

$$
f_{\mathrm{OU}}(t)=\kappa^{2}\left(\frac{t}{\vartheta}-1+e^{-t / \vartheta}\right),
$$

and the effective evolution equation would read

$$
\dot{\rho}_{\mathrm{OU}}(t)=\left(-i \mathcal{L}-\frac{\kappa^{2}}{\vartheta}\left(1-e^{-t / \vartheta}\right) \mathcal{L}^{2}\right) \rho_{\mathrm{OU}}(t),
$$

which indicates that there is a transient effect up to times of the order of the correlation time for the process $\vartheta$. Notice that at $t=0$ there are no non-Liouvillian terms, due to the specific character of the transient of the correlation function. As we shall see, this will be particularly relevant in Zeno's effect. After times of the order of $\vartheta$, the evolution is dictated by the master equation (1).

\section{THE ZENO EFFECT}

The generality of Zeno's effect has been shown in many forms. An early clear description of the general character of the effect was provided by Chiu, Sudarshan, and Misra [8]: if the Hamiltonian is bounded from below, and the initial state is such that the expectation value of the Hamiltonian is finite, then the derivative of the survival probability with respect to time at the initial instant is zero, which entails that the decay of the survival probability is slower than any exponential. The survival probability at ideal time $s$ is given by $\operatorname{Tr}\{\rho(0)[\exp (-i s \mathcal{L}) \rho(0)]\}$. As, under these conditions, this survival probability has no linear term, close to $s=0$, on performing sufficiently frequent measurements we find that the survival probability at any later time is equal to 1: the system is confined to its initial state. On the other hand, if the survival probability did indeed have a linear term in $s$, then the evolution under frequent measurements would be an ex- 
ponential decay, with the decay constant given by the coefficient of the linear term.

We shall now investigate whether the uncertainties in time do eliminate Zeno's effect from taking place. The quantity we want to investigate is the survival probability, that is, given an initial state $\rho(0)$, we should compute

$$
p(t)=\operatorname{Tr}[\rho(0) \rho(t)],
$$

where we measure the time elapsed with a real clock, and, therefore [see Eq. (6)]

$$
p(t)=\operatorname{Tr}\{\rho(0)[\Pi(t,-\mathcal{L}) \rho(0)]\} .
$$

Note that the objects which include the Liouvillian are superoperators, not operators. This entails a slight complication of notation, which we will fix by requiring that superoperators act on everything on their right.

For measurements of time intervals that satisfy the stationarity constraint (3), the linear term in clock time of the survival probability is given by

$$
\frac{1}{\lambda} \operatorname{Tr}\{\rho(0) \ln [g(-\mathcal{L})] \rho(0)\},
$$

which, in terms of the energy basis, can be written as

$$
\frac{2}{\lambda} \sum_{n>m}\left|\rho_{n m}(0)\right|^{2} \ln \left|g\left(E_{n}-E_{m}\right)\right| .
$$

Unless this quantity is zero, the quantum Zeno effect will not take place [notice by the way in the previous computation that, by construction, $g(k)^{*}=g(-k)$ for real $k$.].

Let us assume the validity of the expansion in Eq. (10), with $\langle s\rangle=t$, and further that the initial state is a pure state, $\rho(0)=|a\rangle\langle a|$. In such a situation, the linear term will have a leading term of the form

$$
-\frac{\Delta s^{2}}{2 t} \operatorname{Tr}\left[\rho(0) \mathcal{L}^{2} \rho(0)\right]=-\frac{\Delta s^{2}}{t}(\Delta H)^{2},
$$

where $(\Delta H)^{2}=\left\langle a\left|H^{2}\right| a\right\rangle-\langle a|H| a\rangle^{2}$. That is to say, the small time survival probability will be

$$
p(t)=1-\frac{\Delta s^{2}}{\tau_{Z}^{2}}+\cdots,
$$

with $\tau_{Z}=1 / \Delta H$. No Zeno effect survives: frequent measurements of a system will not maintain it in the initial state, since $\Delta s^{2} \sim t$. There will be an exponential decay no matter how fast the measurements are. Admittedly with a very small decay constant, but exponential nonetheless. We can reobtain this result in a slightly different fashion, by noting that all terms of the Taylor expansion of the (ideal) survival probability in ideal time $s$ contribute to a linear term in $t$ when we perform the averaging with $P(t, s)$ : all cumulants are proportional to $t$, as we have seen, and this is itself a consequence of the stationarity property.

For general clocks, that do not necessarily fulfill stationarity condition (3), the term linear in time will be of the form
$\operatorname{Tr}\left[\rho(0) \partial_{t} \Pi(0,-\mathcal{L}) \rho(0)\right]$, generically nonvanishing. In the Gaussian case, which is not stationary in the sense of Eq. (3), with initial pure state, and the function $f(t)$ defined according to Eq. (14) leads to

$$
p_{\mathrm{G}}(t)=1-\frac{2 \dot{f}(0)}{\tau_{Z}^{2}} t+O\left(t^{2}\right) .
$$

Thus, a very special situation is associated with the OrnsteinUhlenbeck clock, for which $\dot{f}(0)=0$, and Zeno's effect survives. Note, however, the exceptional character of this case, due to the specific form of the transient.

We have thus shown that clock errors generically wash out any possible Zeno effect. This being the case, how can we explain the experimental results of Itano et al. [9]? Forgoing an analysis in terms of the full three-level system coupled to the electromagnetic field [24-28] and neglecting optical pumping due to the measuring laser, we can concentrate on the following conceptual setup: consider a two-level system undergoing a $\pi$-pulse Rabi oscillation. At regular intervals the system is queried as to the state it is in, whether the first or the second level. Formally, at regular intervals the coherences of the density matrix, $\rho_{12}$ and $\rho_{21}$ are set to zero. In the standard analysis, the evolution between measurements is ordinary unitary Schrödinger evolution with the Hamiltonian $H=(\Omega / 2)\left(\begin{array}{ll}0 & 1 \\ 0 & 0\end{array}\right)$. Given an initial density matrix $\rho(0)=\left(\begin{array}{cc}b & 0 \\ 0 & 1-b\end{array}\right)$, and measurements at intervals $\pi /(n \Omega)$, the probability of finding the system in the second level $\left(\begin{array}{l}0 \\ 1\end{array}\right)$ after time $\pi / \Omega$ is

$$
p_{2}\left(\frac{\pi}{\Omega}\right)=\frac{1}{2}+\left(\frac{1}{2}-b\right) \cos ^{n}\left(\frac{\pi}{n}\right) .
$$

If the evolution and measurement process were to take place according to a clock with characteristic function $\Pi(t, k)$, the population of the second level would read

$$
p_{2}\left(\frac{\pi}{\Omega}\right)=\frac{1}{2}+\left(\frac{1}{2}-b\right) \mathcal{C}^{n}\left(\frac{\pi}{n}\right)
$$

where

$$
\mathcal{C}(t)=\frac{1}{2}[\Pi(t, \Omega)+\Pi(t,-\Omega)] .
$$

It is worth mentioning that in the case of a perfect clock, for which $\Pi_{\text {perfect }}(t, k)=\exp (i k t)$ both expressions coincide, as they should.

In order to illustrate this result numerically, let us consider the characteristic function of the master equation (1), which leads to

$$
p_{2}\left(\frac{\pi}{\Omega}\right)=\frac{1}{2}+\left(\frac{1}{2}-b\right) e^{-\Omega \tau \pi / 2} \cos ^{n}\left(\frac{\pi}{n}\right),
$$

with the parameter $\tau$ being defined as $2 \kappa^{2} / \vartheta$. For the system examined by Itano et al., $\Omega=\pi / 256 \mathrm{~m} \mathrm{~s}^{-1}$ and $\tau$ should be larger than $10^{-5} \mathrm{~s}$ for it to have any noticeable effect. As a matter of fact, $\tau$ should be larger than $10^{-4} \mathrm{~s}$ for the effect to 
compete with optical pumping due to the measuring laser. On the other hand, the coherence times mentioned in [9] $(550 \mathrm{~s})$ lead to $\tau \leqslant 10^{-4} \mathrm{~s}$; additionally, the precisions mentioned for time quantities in their experiment are of the order of $10^{-5} \mathrm{~s}$ or better. It follows that the inhibition of transitions from level 1 to level 2 due to frequent measurement is still in place, even though no "perfect" Zeno effect could take place if the clocks themselves are not perfect.

The observability of the master equation (1) can be achieved by purposefully adding a source of errors to the clocks being used in the laboratory, in a controlled manner. One way to detect its effects would be to perform an experiment similar to the one described above, but using a not perfectly regular clock for the measuring pulses.

\section{EXAMPLES: OSCILLATING AND DECAYING SYSTEMS}

Consider an oscillating system in an initial state $|a\rangle$ evolving under the Hamiltonian $H$,

$$
H=\left(\begin{array}{ccc}
0 & \Omega & 0 \\
\Omega & 0 & K \\
0 & K & 0
\end{array}\right), \quad|a\rangle=\left(\begin{array}{l}
1 \\
0 \\
0
\end{array}\right) .
$$

It is easy to compute the exact survival probability under unitary evolution with the preceding Hamiltonian, and then perform the averaging over clock errors, leading to

$$
\begin{aligned}
p(t)= & \frac{1}{\Omega^{\prime 4}}\left\{K^{4}+\frac{\Omega^{4}}{2}+2 K^{2} \Omega^{2} \operatorname{Re}\left[\Pi\left(t, \Omega^{\prime}\right)\right]\right. \\
& \left.+\frac{\Omega^{4}}{2} \operatorname{Re}\left[\Pi\left(t, 2 \Omega^{\prime}\right)\right]\right\},
\end{aligned}
$$

where $\Omega^{\prime}=\sqrt{\Omega^{2}+K^{2}}$.

The small time expansion of this exact evolution in the case of the decohering master equation (1), with $\tau$ $=2 \kappa^{2} / \vartheta$, is quite simply

$$
p_{\text {m.e. }}(t) \sim 1-\Omega^{2} \tau t+O\left(t^{2}\right) .
$$

It is immediate to see that there will be no full Zeno effect, since there is a linear term in the expansion.

If we were to use $g_{\mathrm{M}}(k)$, the full result would be

$$
\begin{aligned}
p_{\mathrm{M}}(t)= & \frac{1}{\Omega^{\prime 4}}\left[K^{4}+\frac{\Omega^{4}}{2}+2 K^{2} \Omega^{2} e^{-\left(1-\cos \Omega^{\prime} \tau\right) t / \tau}\right. \\
& \times \cos \left(\frac{t}{\tau} \sin \Omega^{\prime} \tau\right)+\frac{\Omega^{4}}{2} e^{-\left(1-\cos 2 \Omega^{\prime} \tau\right) t / \tau} \\
& \left.\times \cos \left(\frac{t}{\tau} \sin 2 \Omega^{\prime} \tau\right)\right]
\end{aligned}
$$

A particularly interesting aspect of this expression is that whenever $\Omega^{\prime}=2 n \pi / \tau$, the Zeno effect reappears: the motion of the system is indeed frozen, as Milburn pointed out for different reasons.
Finally, for good Ornstein-Uhlenbeck clocks, we find

$$
\begin{aligned}
p_{\mathrm{OU}}(t)= & \frac{1}{\Omega^{\prime 4}}\left(K^{4}+\frac{1}{2} \Omega^{4}+2 K^{2} \Omega^{2} e^{-\kappa^{2} \Omega^{\prime 2}\left(t / \vartheta-1+e^{-t / \vartheta}\right)}\right. \\
& \times \cos \left(\Omega^{\prime} t\right)+\frac{1}{2} \Omega^{4} e^{-4 \kappa^{2} \Omega^{\prime 2}\left(t / \vartheta-1+e^{-t / \vartheta}\right)} \\
& \left.\times \cos \left(2 \Omega^{\prime} t\right)\right) .
\end{aligned}
$$

As soon as $t$ is substantially bigger than the correlation time of the OU clock, the two survival probabilities computed with the master equation and with the OU probability weight coincide. However, for small times their behavior is radically different: there is no linear term in $t$ in the expansion of $p_{\mathrm{OU}}(t)$, in keeping with the general formal result presented above.

Let us now perform an analogous computation for a model of a decaying system, in such a way that we can extrapolate without difficulty to more general decaying processes. On the by side, we will obtain an expression for the line shape, confirming generically the result of Adler's that it is not modified by phase decoherence [17].

Consider thus a system with a discrete orthogonal basis $\{|a\rangle\} \cup\{|\omega\rangle\}_{\omega}$, where $\omega$ takes values in some discrete set of frequencies, and such that the Hamiltonian can be written as $H=H_{0}+V$, with

$$
H_{0}=\omega_{a}|a\rangle\left\langle a\left|+\sum_{\omega} \omega\right| \omega\right\rangle\langle\omega|,
$$

and the only nonzero elements of $V$ being $\langle a|V| \omega\rangle$ and their complex conjugates. The initial state will be the pure state $|a\rangle$. This system is a simplified model of decay from this pure state to the rest of Hilbert space.

By using the resolvent, $G(z)=(z-H)^{-1}$, the exact Dyson-Schwinger's equation

$$
G(z)=G_{0}(z)+G_{0}(z) V G(z)
$$

for this system can be solved to

$$
\begin{gathered}
G_{a}(E)=\frac{1}{E-\omega_{a}-\Sigma_{a}(E)}, \\
G_{\omega}(E)=\frac{\langle\omega|V| a\rangle}{(E-\omega)\left[E-\omega_{a}-\Sigma_{a}(E)\right]},
\end{gathered}
$$

where

$$
G_{a}(z)=\langle a|G(z)| a\rangle, \quad G_{\omega}(z)=\langle\omega|G(z)| a\rangle
$$

and

$$
\Sigma_{a}(E)=\sum_{\omega} \frac{|\langle a|V| \omega\rangle|^{2}}{E-\omega}
$$

is the exact self-energy for this model. In more general models, it is still the case that we can write the expectation value 
of the resolvent in state $|a\rangle$ in terms of the self-energy $\Sigma_{a}(E)$, as expressed in Eq. (17). The change will come about because of the modifications of the self-energy, which will no longer be determined by Eq. (19). The transition quantity $G_{\omega}(E)$ will also have a different expression.

The small time behavior of the survival amplitude in ideal time $s$ is determined by the large-energy behavior of $G_{a}(E)$ and, consequently, of the large energy behavior of the selfenergy. In our specific example, it is very easy to see that, for energies large in comparison to all $\omega$ values,

$$
\begin{aligned}
\Sigma_{a}(E) & \approx \frac{1}{E} \sum_{\omega}|\langle a|V| \omega\rangle|^{2} \\
& =\frac{1}{E}\left(\left\langle a\left|H^{2}\right| a\right\rangle-\langle a|H| a\rangle^{2}\right)=\frac{1}{\tau_{Z}^{2} E} .
\end{aligned}
$$

Under general assumptions, and for large energies, the selfenergy function has exactly the same structure $\Sigma_{a}(E)$ $\sim 1 / \tau_{Z}^{2} E$ for generic models. Then, since the survival probability amplitude $\mathcal{A}(s)$ is determined by the resolvent and hence by the self-energy through

$$
\mathcal{A}(s)=\frac{i}{2 \pi} \int_{\Gamma} d E e^{-i E s} G_{a}(E),
$$

where by $\Gamma$ here we denote the adequate integration path, the survival probability at small ideal time $s$ is [11]

$$
p_{\text {ideal }}(s)=|\mathcal{A}(s)|^{2} \sim 1+\frac{2}{4+\omega_{a}^{2} \tau_{Z}^{2}}\left[\cos \left(\sqrt{4+\omega_{a}^{2} \tau_{Z}^{2}} \frac{s}{\tau_{Z}}\right)-1\right] .
$$

On performing the averages for Eq. (20), we obtain

$$
p(t) \sim 1+\frac{2}{4+\omega_{a}^{2} \tau_{Z}^{2}}\left[\operatorname{Re} \Pi\left(t, \sqrt{\omega_{a}^{2}+4 / \tau_{Z}^{2}}\right)-1\right] .
$$

The objection might be posed that small $t$ does not imply that $s$ is small (the comparison term in order to state the smallness or otherwise of these dimensionful quantities is always taken to be the characteristic evolution time of the system). However, we have seen above that clocks that satisfy the stationarity requirement of Eq. (3) display an average value of $s$ that tracks $t$, and that the dispersion is given as $\Delta s^{2}$ $\sim t$. It then follows that indeed we can approximate small $t$ behavior by extracting the small $s$ behavior and then averaging.

We see again that through this method we recover again that there is a linear term, and that the disappearance of Zeno's effect is completely generic. An exception can be found in the OU clock case, for which

$$
p_{\mathrm{OU}}(t) \sim 1-\frac{t^{2}}{\tau_{Z}^{2}},
$$

as we already know.
We shall now study the large time behavior of the system. Assume now that the states orthogonal to the initial one, $|a\rangle$, form a continuum, in the manner postulated by Weisskopf and Wigner [29], so that an imaginary part can arise for the poles of $G_{a}(E)$. Then, for large times, the dominant behavior, both for the survival amplitude and for the probability amplitude to find the system in a state different from the initial one, will be determined by the pole of $G_{a}(E)$ closest to the real axis. Assume there is only one such relevant pole, of the form $\omega_{p}-i \gamma / 2$. The contribution of this simple pole to the survival amplitude is

$$
\mathcal{A}_{\mathrm{p}}(s)=\sqrt{\mathcal{Z}_{\mathrm{p}}} e^{-i \omega_{p} s-\gamma s / 2},
$$

where $\mathcal{Z}_{\mathrm{p}}$ is the relevant residue. Hence, the contribution of the simple pole to the survival probability at ideal time $s$ becomes

$$
p_{\text {p,ideal }}(s)=\mathcal{Z}_{\mathrm{p}} e^{-\gamma s} .
$$

If this were the only contribution to the survival probability, it would be immediate to conclude that at clock time $t$ the survival probability would read

$$
p_{\mathrm{p}}(t)=\mathcal{Z}_{\mathrm{p}} \Pi(t, i \gamma) .
$$

For large times, it is to be expected that the main contribution of real clocks will be given by the master equation approximation. It results that

$$
p_{\mathrm{p}}(t) \approx \mathcal{Z}_{\mathrm{p}} e^{-\gamma(1-\gamma \tau / 2) t},
$$

which means that the long-times delay is slowed down as an effect of clock errors, the new decay constant being given by $\gamma(1-\gamma \tau / 2)$. As the half-life and the error dispersion parameter approach, the long-time decay will become slower.

Under the same approximation (single simple pole or Weisskopf-Wigner's approximation), we have for the probability amplitude of states orthogonal to the initial one, at very late times, and for the specific Hamiltonian (16),

$$
\mathcal{B}_{\omega}(s)=\left\langle\omega e^{-i s H} \mid a\right\rangle \sim \frac{\langle\omega|V| a\rangle\left(e^{-i \omega_{p} s} e^{-\gamma s / 2}-e^{-i \omega s}\right)}{\omega_{p}-\omega-i \gamma / 2},
$$

whence it follows that the probability of finding state $|\omega\rangle$ at (sufficiently large) clock time $t$ is

$$
\begin{aligned}
p_{\omega}(t)= & \left|\mathcal{B}_{\omega}(t)\right|^{2} \\
= & \theta(t) \frac{|\langle\omega|V| a\rangle|^{2}}{\left(\omega_{p}-\omega\right)^{2}+\gamma^{2} / 4} \\
& \times\left[1+\Pi(t, i \gamma)-\Pi\left(t, \omega-\omega_{p}+i \gamma / 2\right)\right. \\
& \left.-\Pi\left(t, \omega_{p}-\omega+i \gamma / 2\right)\right] \\
\rightarrow & \frac{|\langle\omega|V| a\rangle|^{2}}{\left(\omega_{p}-\omega\right)^{2}+\gamma^{2} / 4},
\end{aligned}
$$

which means that the line shape, within the approximation carried out (Weisskopf-Winger approximation, see Ref. [29]) 
does not change at all because of the errors in the clocks. We have not actually fully proved this statement: we have not justified that the use of the long-time approximation in ideal time is enough before taking the average. However, for probability densities that lead to condition (3), the average value of $s$ is proportional to $t$, and its quadratic dispersion goes instead with $\sqrt{t}$. It follows that one can carry over the approximation of large ideal times to large Schrödinger time.

\section{CONCLUSIONS}

We have shown the following using a general description of errors in clocks:

(1) Decoherence, although a slow process if the clocks are good, starts taking place immediately for generic models of clocks.

(2) As a consequence, Zeno's effect would never fully freeze the system in its initial state, for generic clocks.

(3) We have found two kinds of exception to this result; whenever the decoherence itself freezes the state, as happens for some frequencies in the case put forward by Milburn, and in the Ornstein-Uhlenbeck clock.

(4) Under the assumptions of real clocks put forward in Ref. [14] and in this paper, there is no change in the line shape due to nonlocality in time.

The master equation (1) has also been shown to be a generic approximation to evolutions under clocks with different sets of errors, in a controlled manner, thus amenable to measurement.

\section{ACKNOWLEDGMENTS}

This work has been supported by Ministerio de Ciencia y Tecnologí a (Grant No. AEN99-0135, fBFM2000-0816-C0303, BFM2001-0213, BFM2002-04031-C02-02, FPA200202037), UPV-EHU (Grant No. 00039.310-13507/2001), and the Basque Government (Grant No. PI-1999-28). I.L.E. is grateful to M. A. Valle, J. L. Mañes, and J. G. Muga for useful comments.
[1] Time in Quantum Mechanics, edited by J. G. Muga, R. Sala Mayato, and I. L. Egusquiza, Lecture Notes in Physics, Monographs, Vol. 2 (Springer, Berlin, 2002).

[2] B. Misra and E.C.G. Sudarshan, J. Math. Phys. 18, 756 (1977).

[3] G.R. Allcock, Ann. Phys. (N.Y.) 53, 253 (1969).

[4] G.R. Allcock, Ann. Phys. (N.Y.) 53, 286 (1969).

[5] G.R. Allcock, Ann. Phys. (N.Y.) 53, 311 (1969).

[6] L.A. Khalfin, Pis'ma Zh. Eksp. Teor. Fiz. 8, 106 (1968) [JETP Lett. 8, 65 (1968)].

[7] R.G. Winter, Phys. Rev. 123, 1503 (1961).

[8] C.B. Chiu, E.C.G. Sudarshan, and B. Misra, Phys. Rev. D 16, 520 (1977).

[9] W.M. Itano, D.J. Heinzen, J.J. Bollinger, and D.J. Wineland, Phys. Rev. A 41, 2295 (1990).

[10] D. Home and M.A.B. Whitaker, Ann. Phys. (N.Y.) 258, 237 (1997).

[11] P. Facchi and S. Pascazio, e-print quant-ph/0207030.

[12] G.J. Milburn, Phys. Rev. A 44, 5401 (1991).

[13] G.C. Ghirardi, A. Rimini, and T. Weber, Phys. Rev. D 34, 470 (1986).

[14] I.L. Egusquiza, L.J. Garay, and J.M. Raya, Phys. Rev. A 59, 3236 (1999).
[15] R. Bonifacio, Nuovo Cimento Soc. Ital. Fis., B 114B, 473 (1999).

[16] H. Carmichael, An Open Systems Approach To Quantum Optics (Springer-Verlag, Berlin, 1993).

[17] S.L. Adler, Phys. Rev. D 67, 025007 (2003).

[18] P. Facchi, H. Nakazato, and S. Pascazio, Phys. Rev. Lett. 86, 2699 (2001).

[19] W. Królikowski hep-th/0212232.

[20] D. Salgado and J.L. Sánchez-Gómez, e-print quant-ph/0212042.

[21] R.K. Gainutdinov, J. Phys. A 32, 5657 (1999).

[22] R.K. Gainutdinov, A.A. Mutygullina, and W. Scheid, e-print quant-ph/0210144.

[23] P. Busch, e-print quant-ph/9909073.

[24] S.L. Braunstein and G.J. Milburn, Phys. Rev. A 51, 1820 (1995).

[25] L.E. Ballentine, Phys. Rev. A 43, 5165 (1991).

[26] E. Block and P.R. Berman, Phys. Rev. A 44, 1466 (1991).

[27] V. Frerichs and A. Schenzle, Phys. Rev. A 44, 1962 (1991).

[28] A. Beige and G.C. Hegerfeldt, Phys. Rev. A 53, 53 (1996).

[29] V. Weisskopf and E. Wigner, Z. Phys. 63, 54 (1930). 